\title{
Augmented reality and physical hybrid model simulation for preoperative planning of metopic craniosynostosis surgery
}

\author{
Giselle Coelho, MD, PhD, ${ }^{1-4}$ Nicollas Nunes Rabelo, MD, ${ }^{3}$ Eduardo Vieira, MD, ${ }^{1}$ Kid Mendes, MD, ${ }^{5}$ \\ Gustavo Zagatto, MD, ${ }^{3}$ Ricardo Santos de Oliveira, MD, PhD, ${ }^{6}$ \\ Cassio Eduardo Raposo-Amaral, MD, PhD, ${ }^{7}$ Maurício Yoshida, MD, ${ }^{1,4}$ \\ Matheus Rodrigues de Souza, MD, ${ }^{8}$ Caroline Ferreira Fagundes, MD, ${ }^{8}$ \\ Manoel Jacobsen Teixeira, MD, $\mathrm{PhD},{ }^{3}$ and Eberval Gadelha Figueiredo, MD, $\mathrm{PhD}^{3}$
}

\begin{abstract}
${ }^{1}$ Santa Marcelina Hospital; 2EDUCSIM Institute; ${ }^{3}$ University of São Paulo, USP; and ${ }^{4}$ Sabará Children's Hospital, São Paulo, Brazil; ${ }^{5}$ State University of Amazonas, Manaus, Brazil; ${ }^{6}$ Division of Neurosurgery, School of Medicine of Ribeirao Preto, University of São Paulo Ribeirao Preto, São Paulo, Brazil; ${ }^{7}$ Craniofacial Surgery Institute of the Hospital SOBRAPAR, Campinas, Brazil; and ${ }^{8}$ University of the State of Mato Grosso, Brazil
\end{abstract}

\begin{abstract}
OBJECTIVE The main objective of neurosurgery is to establish safe and reliable surgical techniques. Medical technology has advanced during the 21st century, enabling the development of increasingly sophisticated tools for preoperative study that can be used by surgeons before performing surgery on an actual patient. Laser-printed models are a robust tool for improving surgical performance, planning an operative approach, and developing the skills and strategy to deal with uncommon and high-risk intraoperative difficulties. Practice with these models enhances the surgeon's understanding of 3D anatomy but has some limitations with regard to tactile perception. In this study, the authors aimed to develop a preoperative planning method that combines a hybrid model with augmented reality (AR) to enhance preparation for and planning of a specific surgical procedure, correction of metopic craniosynostosis, also known as trigonocephaly.

METHODS With the use of imaging data of an actual case patient who underwent surgical correction of metopic craniosynostosis, a physical hybrid model (for hands-on applications) and an AR app for a mobile device were created. The hybrid customized model was developed by using analysis of diagnostic CT imaging of a case patient with metopic craniosynostosis. Created from many different types of silicone, the physical model simulates anatomical conditions, allowing a multidisciplinary team to deal with different situations and to precisely determine the appropriate surgical approach. A real-time AR interface with the physical model was developed by using an AR app that enhances the anatomic aspects of the patient's skull. This method was used by 38 experienced surgeons (craniofacial plastic surgeons and neurosurgeons), who then responded to a questionnaire that evaluated the realism and utility of the hybrid AR simulation used in this method as a beneficial educational tool for teaching and preoperative planning in performing surgical metopic craniosynostosis correction.
\end{abstract}

RESULTS The authors developed a practice model for planning the surgical cranial remodeling used in the correction of metopic craniosynostosis. In the hybrid AR model, all aspects of the surgical procedure previously performed on the case patient were simulated: subcutaneous and subperiosteal dissection, skin incision, and skull remodeling with absorbable miniplates. The pre- and postoperative procedures were also carried out, which emphasizes the role of the AR app in the hybrid model. On the basis of the questionnaire, the hybrid AR tool was approved by the senior surgery team and considered adequate for educational purposes. Statistical analysis of the questionnaire responses also highlighted the potential for the use of the hybrid model in future applications.

CONCLUSIONS This new preoperative platform that combines physical and virtual models may represent an important method to improve multidisciplinary discussion in addition to being a powerful teaching tool. The hybrid model associated with the AR app provided an effective training environment, and it enhanced the teaching of surgical anatomy and operative strategies in a challenging neurosurgical procedure.

https://thejns.org/doi/abs/10.3171/2019.12.FOCUS19854

KEYWORDS augmented reality; hybrid model simulation; craniosynostosis; preoperative planning; neurosurgery 
$\mathrm{M}$ ETOPIC craniosynostosis, also termed trigonocephaly, is one of the most common types of craniosynostosis. Formed by premature closure of the metopic suture, ${ }^{6,13,14}$ metopic craniosynostosis represents approximately $23 \%-28 \%$ of all craniosynostosis cases. ${ }^{13,14}$ In the literature, there is uncertainty about the normal age of fusion of the metopic suture. ${ }^{14}$ Most authors report that the metopic suture begins to close after the first year and is obliterated by 7-8 years of age, after a period of major broadening of the forehead. ${ }^{11,15,17}$

Many simulators have been developed as a first step to enhance the development of the surgical skills of residents in plastic surgery and neurosurgery. ${ }^{2}$ Cadaveric sheep craniums were previously used as a simulator, but this method has clear limitations, ${ }^{8}$ and animal models may raise ethical objections and their use is even forbidden in some countries. ${ }^{3}$ Besides that, with complex and high-risk procedures such as craniosynostosis surgery in infants, the opportunities for hands-on training have been very limited.

The availability of 3D printing technology has expanded the possibilities for developing alternative solutions to this problem through the use of lifelike models to increase understanding of normal and pathological anatomy. 3D models are being used in medicine for many applications, including surgical planning, patient education and procedure rehearsal, and trainee education. ${ }^{16}$

The process of creating 3D models for use in surgical training begins with segmentation of image data to convert the anatomical imaging information obtained by CT scanning and MRI into a 3D patient-specific digital model of all relevant anatomical structures. ${ }^{2,16}$

Augmented reality (AR) transforms virtual objects into lifelike video images in real time, providing the neurosurgeon with specific anatomical information and elaborated metrics. ${ }^{10,11}$ With recent advancements, several systems have been proposed for surgical planning that enable the surgeon to be engaged with both the graphic AR representation and the actual surgical field simultaneously. $7,9,10$ Despite the importance of improving the technical skills, attitudes, and interpersonal abilities of those who perform surgical procedures, few studies have examined the use of AR technology in preoperative planning to determine the most favorable surgical approaches. ${ }^{10}$ With the development of an AR-based training simulator, surgeons in training can experience realistic simulations of surgical operations comparable to those encountered in actual neurosurgery. ${ }^{11}$

Here we present a novel hybrid modality that combines AR with a physical hybrid model specifically for training and preoperative planning of metopic craniosynostosis correction.

\section{Methods}

This research study was divided into 3 parts. The first part involved the design and fabrication of a synthetic physical hybrid model of metopic craniosynostosis using data from an actual patient. The second part consisted of the development of the AR model, and the third part included the testing and evaluation of the AR model, such as the model's realism, applicability in preoperative planning, and potential teaching content, by senior neurosur-
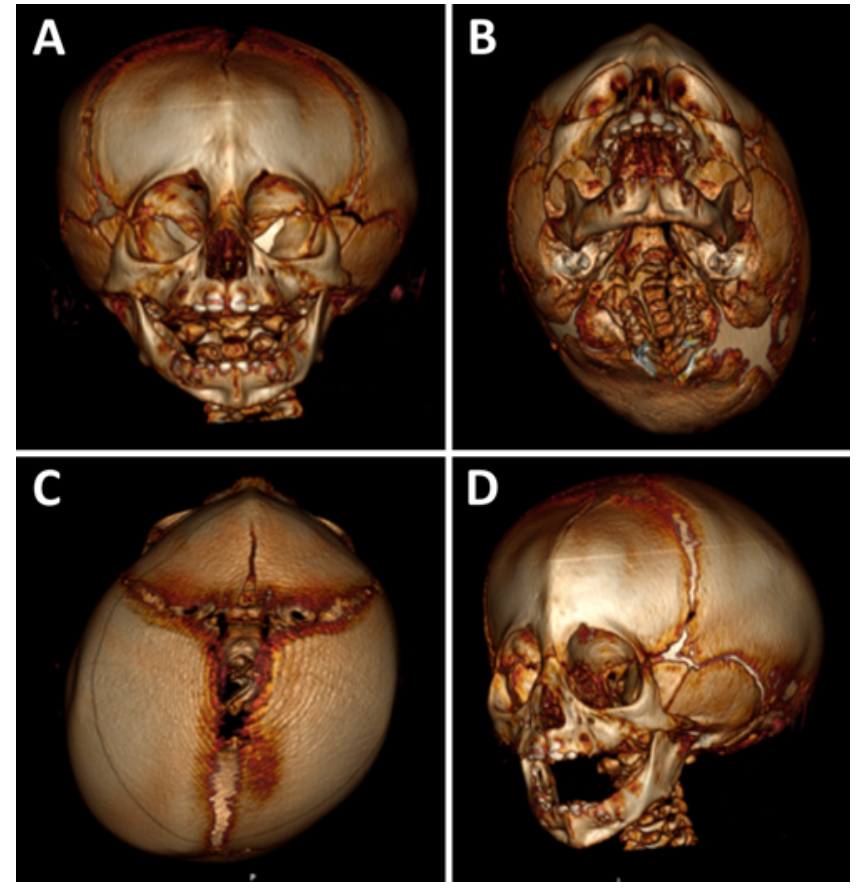

FIG. 1. A-D: 3D reconstructions created from CT imaging studies of the case patient with metopic craniosynostosis.

geons and craniofacial plastic surgeons who participated in an annual Brazilian craniofacial course held at the São Paulo Medtronic Headquarters Laboratory.

The questionnaire was completed by 38 senior surgeons, $13(34.2 \%)$ craniofacial plastic surgery and 25 (65.8\%) neurosurgery experts. The survey content was applicable to the experience of the experts, who evaluated the planning method, which used the hybrid model together with 3DSlicer and InVesalius software. Most respondents agreed that it would be possible to perform the diagnosis from the simulator $(\mathrm{p}=0.01)$, and more than $50 \%$ agreed the simulation provided a reliable representation of the skull of a real patient $(\mathrm{p}=0.03)$.

\section{Statistical Analysis}

All analyses were performed using IBM SPSS version 20.0 (IBM Corp.). Values of $\mathrm{p} \leq 0.05$ were deemed statistically significant. Cronbach's alpha was applied to assess internal reliability according to a very high classification score (Cronbach's alpha $=0.94)$.

\section{Case Presentation and Planning Method}

The case patient was a 5-month-old boy who presented with a diagnosis of metopic craniosynostosis (Fig. 1). CT imaging data were acquired, and a 3D reconstruction of the images was performed (Fig. 2) for use in the development of the AR model and the physical hybrid model used for planning the surgery in the case patient.

\section{Description of the New Preoperative Planning Modality}

Part I: Development of the Metopic Craniosynostosis Model

The first step of the development process consisted 


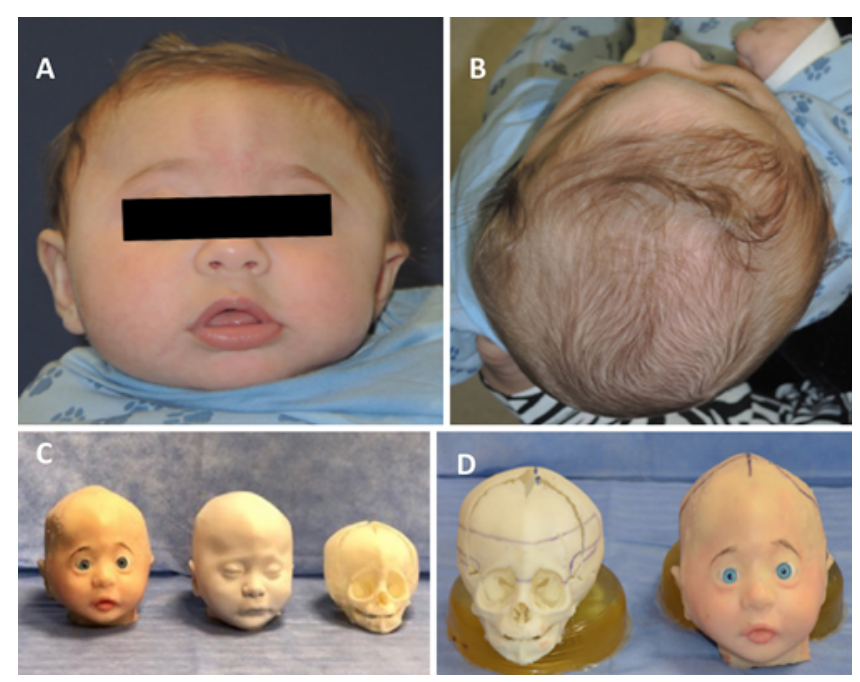

FIG. 2. A and B: Metopic craniosynostosis preoperative images. $C$ and D: The hybrid AR-physical model combines the CT imaging reconstructions and the 3D-printed models of skin and skull pieces.

of obtaining the 3D-printed skull model. After that, the model was improved according to a handmade process, with different materials. This model was built with a special resin and some types of silicone with thermosensitive properties and presented some human tissue characteristics, including textures, consistencies, and mechanical resistance.

The anatomical aspects of the skull were based on CT DICOM thin-slice images of a real patient (the 5-monthold male infant case patient), which allowed 3D reconstruction. The material was composed of high-performance platinum-cured liquid silicone components, which are used in many applications ranging from creating skin effects to making different tissues. An infinite number of color effects may be achieved by adding silicone pigments and/or effect powders. The cured rubber could also be painted. The cured material used is safe and certified by an independent laboratory (ISO 10993-10; Dragon Skin).

The surgical training using the metopic craniosynostosis model was performed as if it was a real surgery, using the same surgical instruments.

\section{Part II: Development of the Customized AR Model}

The authors, in scientific partnership with the Samsung company (www.samsung.com.br), worked on creating and developing a new AR model that can be used as a cellphone app (Video 1).

VIDEO 1. AR simulator for preoperative surgical planning. Copyright Nicollas Nunes Rabelo, University of São Paulo. Used with permission. Click here to view.

The production of a 3D model for AR for tablets or smartphones is usually based on the requirement to design objects that can be used with a variety of devices with different processing capabilities. In the case of medical uses, for example, for which there is often a demand to present real models of patients, we used software capable of transforming sequences of MRI or CT files into polygonal models. Programs such as 3DSlicer and InVesalius were applied to obtain various types of fabricated materials. The model creation process required the selection and slide-by-slide application of masks in order to eliminate areas that did not matter or to paint in the parts that mattered.

In general, the objects obtained from selection and converted to polygonal mesh have high vertex density, which makes them incompatible for use directly in the development of augmented or virtual reality. Therefore, it is necessary to use 2 possible methods of mesh reduction in order to achieve this outcome. The first method uses the automatic polygon reduction features available in some computer graphics software (such as ZBrush and MeshMixer, among others), which enable the program to considerably decrease the number of triangular polygons on flatter surfaces, keeping as many polygons as possible in curves and edges. The other option is to reconstruct the model using the retopology method, a feature that is also available in specific software programs (e.g., 3DS Max, Maya, and ZBrush). This method requires a lot of manual work by the designer, but it has the advantage of allowing the construction of a more organized mesh model composed of quadrilateral polygons. When the object has a bitmap texture (surface coating), it is essential to ensure that such maps do not contain very high resolutions, because the loading of this information, together with the polygonal object itself, will compromise performance.

The following tools were used: Unity Engine, for application development, and ARCore, Google's development kit for building AR applications.

At the start of the application, white dots are shown, which are used to mark the position of the 3D object. To make this happen, the user needs to look at a flat space, then move the phone from side to side until there is enough space for the projection of the 3D object.

After the positioning of the 3D object, new buttons appear with the following features to visualize the 3D object: button 1, visualize the skin layer; button 2, visualize the skull layer; button 3, visualize the sectioned skull layer. The application has 2 mechanical controls, rotate and zoom the 3D object, to improve the user experience.

\section{Participants and Data Collection}

Part III: Realism and Educational Value of the Craniosynostosis AR Simulator

Thirty-eight participants were recruited from a Brazilian craniofacial course hosted by the Brazilian Pediatric Neurosurgery and Craniofacial Surgery Societies at the Medtronic Laboratory in São Paulo. The participants included expert craniofacial surgeons and neurosurgeons. Standard Galaxy 9 and 10 (Samsung) cellphones with the installed AR app were used.

Following the AR testing, the study participants evaluated the realism of the holographic projection, including the use of the device for teaching purposes. The questionnaire included 15 items, with items focusing on the representation of the patient anatomy and image handling (rotation and zoom properties) and items considering the realism of the model and its teaching value. The surgeons were asked to rate their level of agreement by using a 

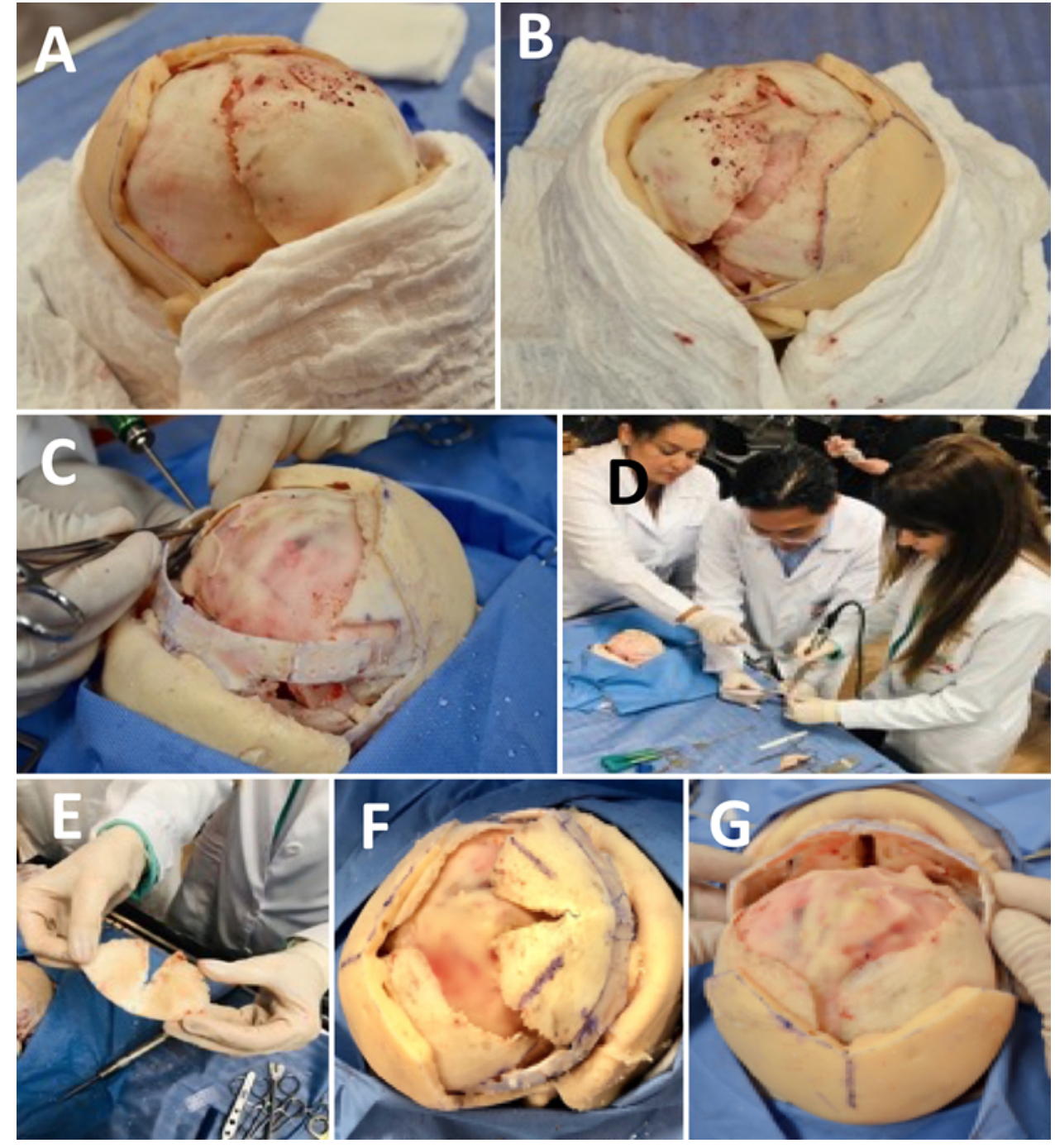

FIG. 3. Preoperative planning using the hybrid model. A: The first step of the development process consisted of obtaining the 3D-printed skull model. B and C: Craniosynostosis craniotomy. D and E: Craniosynostosis hybrid mosaic surgery performed. F and G: Testing the best way of reconstructing the skull.

5-point Likert scale, with possible scores for each item ranging from 1 (strongly disagree) to 5 (strongly agree). The participants were also encouraged to provide free-text comments regarding the device's effectiveness and realism for training and educational purposes in the future.

\section{Results}

The hybrid model allowed a detailed discussion of the aspects of the surgical planning, providing tissues with different tactile and resistance qualities, as well as prediction of the orbit remodeling measurements with excellent precision. It was possible to simulate the surgical approach step-by-step, use real instruments, cut the skin (the silicone mimicked the tissue accurately), perform the osteotomies, and dissect according to plan, with appropriate tactile feedback (Fig. 3). In addition, the model was considered a fundamental and valuable factor in the multidisciplinary discussion between the neurosurgeons and plastic surgeons. Table 1 shows the evaluation results of the AR app. The questionnaire items for the AR app evaluation are shown in Table 2, with the number of answers for each level of agreement, as well as the $p$ values found from the item correlation test.

As planned in the hybrid model, the surgical approach was a frontal craniotomy associated with bilateral orbit bar advancement and absorbable plate reconstruction. The obtained measurements of the bilateral orbit advancement were accurately reproduced during the actual surgery.

The case patient did not present any postoperative complications after undergoing the planned metopic craniosynostosis surgery, and the hospitalization period was 4 days. The patient was also evaluated during the postoperative period. As described by Whitaker et al., ${ }^{18}$ the morphological evaluation for global esthetic results is classified into 4 categories, from I (no refinements or surgical revisions considered desirable or necessary) to IV (a major craniofacial procedure advisable). The final considerations 
TABLE 1. Results of AR app evaluation by neurosurgeons and craniofacial plastic surgeons

\begin{tabular}{ccccccc}
\hline & \multicolumn{6}{c}{ Surgeon Response, $\%$ (no.) } \\
\cline { 2 - 5 } Question No. & Strongly Disagree & Disagree & Neutral & Agree & Strongly Agree & Total Responses \\
\hline 1 & - & - & $5.26 \%(2)$ & $47.37 \%(18)$ & $47.37 \%(18)$ & 38 \\
\hline 2 & - & $7.89 \%(3)$ & $10.53 \%(4)$ & $50.00 \%(19)$ & $31.58 \%(12)$ & 38 \\
\hline 3 & - & $5.26 \%(2)$ & $7.89 \%(3)$ & $34.21 \%(13)$ & $52.63 \%(20)$ & 38 \\
\hline 4 & - & $2.63 \%(1)$ & $15.79 \%(6)$ & $39.47 \%(15)$ & $42.11 \%(16)$ & 38 \\
\hline 5 & - & $2.63 \%(1)$ & $7.89 \%(3)$ & $42.11 \%(16)$ & $47.37 \%(18)$ & 38 \\
\hline 6 & - & - & $2.63 \%(1)$ & $39.47 \%(15)$ & $57.89 \%(22)$ & 38 \\
\hline 7 & - & $2.63 \%(1)$ & $2.63 \%(1)$ & $42.11 \%(16)$ & $52.63 \%(20)$ & 38 \\
\hline 8 & - & $7.89 \%(3)$ & $15.79 \%(6)$ & $47.37 \%(18)$ & $28.94 \%(11)$ & 38 \\
\hline 9 & $2.63 \%(1)$ & $7.89 \%(3)$ & $18.42 \%(7)$ & $47.37 \%(18)$ & $23.68 \%(9)$ & 38 \\
\hline 10 & - & $5.26 \%(2)$ & $13.16 \%(5)$ & $50.00 \%(19)$ & $31.58 \%(12)$ & 38 \\
\hline 11 & - & - & $31.25 \%(10)$ & $50.00 \%(16)$ & $18.75 \%(6)$ & 32 \\
\hline 12 & $2.70 \%(1)$ & $5.41 \%(2)$ & $13.51 \%(5)$ & $43.24 \%(16)$ & $35.14 \%(13)$ & 37 \\
\hline 13 & - & $10.53 \%(4)$ & $23.68 \%(9)$ & $47.37 \%(18)$ & $18.42 \%(7)$ & 38 \\
\hline 14 & - & $2.63 \%(1)$ & $7.89 \%(3)$ & $50.00 \%(19)$ & $39.47 \%(15)$ & 38 \\
\hline 15 & - & $2.63 \%(1)$ & $7.89 \%(3)$ & $50.00 \%(19)$ & $39.47 \%(15)$ & 38 \\
\hline
\end{tabular}

were done by the patient's parents and the surgical team, and the outcome was classified as a good result (Whitaker classification I) (Fig. 4).

It is noteworthy that the clear majority of questionnaire respondents agreed with the simulator's possible contribution to the patient's preoperative planning (totally agree/ agree: $76.31 \%$; $\mathrm{p}=0.004)$. In addition, $73.68 \%$ agreed (mostly strongly agreed) with the possible beneficial effect on the postoperative outcome with the use of the simulator $(\mathrm{p}=0.002)$.

Further, it is essential to consider that the experts highlighted the possibility of manually expanding the object

TABLE 2. AR evaluation items and Likert scale responses by neurosurgeons and craniofacial plastic surgeons

\begin{tabular}{|c|c|c|c|c|c|c|}
\hline \multirow[b]{2}{*}{ Questionnaire Item } & \multicolumn{5}{|c|}{ Surgeon Response } & \multirow[b]{2}{*}{$\begin{array}{c}\mathrm{p} \\
\text { Value }\end{array}$} \\
\hline & $\begin{array}{l}\text { Strongly } \\
\text { Disagree }\end{array}$ & Disagree & Neutral & Agree & $\begin{array}{l}\text { Strongly } \\
\text { Agree }\end{array}$ & \\
\hline 1. It was possible to diagnose the patient using this simulator & 0 & 0 & 1 & 18 & 19 & 0.01 \\
\hline 2. This virtual simulator provides a reliable cranial representation of a real patient & 0 & 3 & 4 & 12 & 19 & 0.03 \\
\hline 3. I would participate in future preoperative planning sessions with this simulator & 0 & 2 & 3 & 20 & 13 & 0.02 \\
\hline $\begin{array}{l}\text { 4. I believe practicing with this simulator will improve my ability to define the best surgical } \\
\text { access }\end{array}$ & 0 & 1 & 6 & 16 & 15 & 0.014 \\
\hline $\begin{array}{l}\text { 5. I believe that the 3D visualization of the anatomical aspects of the simulator is an ap- } \\
\text { propriate tool for multidisciplinary discussions }\end{array}$ & 0 & 0 & 4 & 18 & 16 & 0.02 \\
\hline 6. I believe that AR may contribute to the patient (or family) understanding the pathology & 0 & 0 & 1 & 22 & 15 & 0.00 \\
\hline $\begin{array}{l}\text { 7. I believe that AR may contribute to the patient (or family) understanding the surgery } \\
\text { that will be performed }\end{array}$ & 0 & 1 & 1 & 20 & 16 & 0.001 \\
\hline 8. Using the AR virtual simulator as a preoperative planning tool is extremely useful & 0 & 3 & 6 & 10 & 19 & 0.004 \\
\hline 9. Preoperative planning with this simulator can improve postoperative outcome & 1 & 3 & 6 & 9 & 19 & 0.002 \\
\hline 10. The 3D reconstruction from real images was very realistic & 0 & 2 & 5 & 12 & 19 & 0.006 \\
\hline 11. The metopic craniosynostosis video was very realistic & 0 & 0 & 10 & 16 & 6 & 0.029 \\
\hline 12. The spatial orientation of the tissues (skin and skull) was realistic & 1 & 2 & 6 & 15 & 13 & 0.015 \\
\hline 13. It was possible to visualize skull base changes accurately & 0 & 4 & 9 & 17 & 8 & 0.012 \\
\hline $\begin{array}{l}\text { 14. The possibility of manually enlarging the object can help in understanding the pathol- } \\
\text { ogy and discussing surgical access }\end{array}$ & 0 & 1 & 3 & 19 & 15 & 0.016 \\
\hline $\begin{array}{l}\text { 15. The possibility of manually rotating the object can help in understanding the pathology } \\
\text { and discussing surgical access }\end{array}$ & 0 & 1 & 3 & 17 & 5 & 0.008 \\
\hline
\end{tabular}




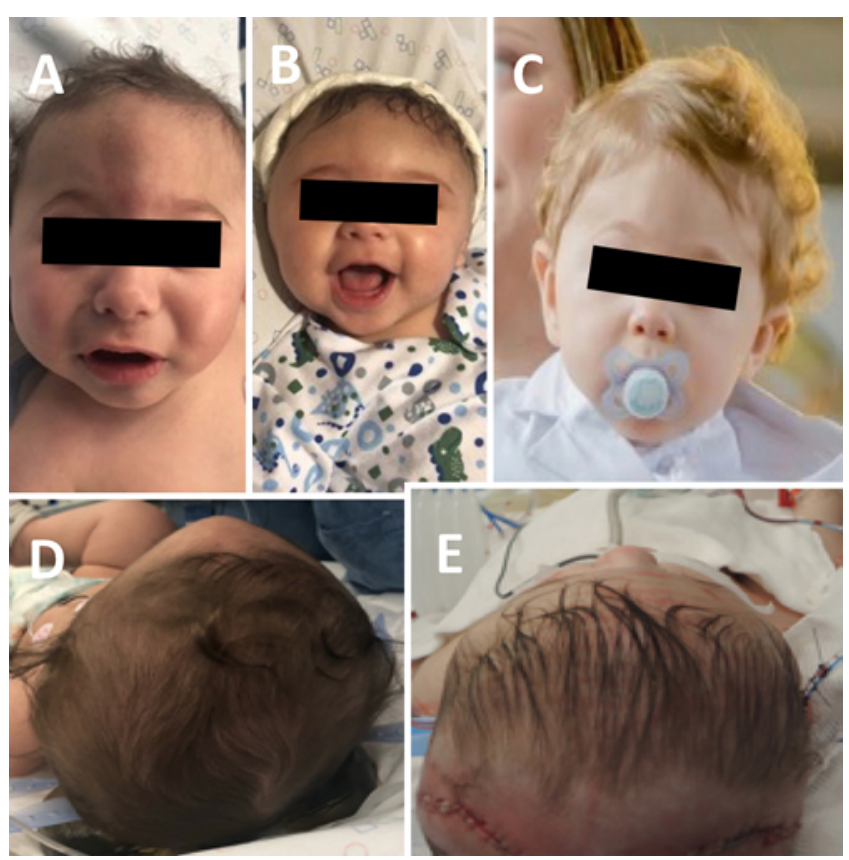

FIG. 4. Images of the case patient. A: Preoperative image (frontal view). B: Immediately postoperative image. C: Follow-up image at 7 months. D: Preoperative image (superior view). E: Immediately postoperative image (superior view).

using the zoom feature, which would improve understanding of the pathology and the discussion of surgical access (totally agree/agree: $87.17 \% ; \mathrm{p}=0.016$ ).

\section{Discussion}

Early fusion of one or more cranial sutures may result in structural abnormalities in head shape, visual deficits, intracranial hypertension, and neurodevelopmental complications..$^{13}$ Metopic craniosynostosis, also termed trigonocephaly, is craniosynostosis characterized by a triangular forehead shape, hypotelorism, lateral orbital retrusion, bitemporal narrowing, and compensatory occipitoparietal expansion. ${ }^{13,14}$ Studies have revealed a wide range in the prevalence of intracranial hypertension in patients with isolated trigonocephaly (0\%-33\%), although screening is difficult in this patient population, given the low sensitivity of funduscopic examination and imaging in newborns and children. ${ }^{1,4}$ These children also frequently have developmental delays in speech and language, as well as cerebral function disorders, particularly dysfunction of the frontal lobes. ${ }^{19}$

Standard care for these patients is surgical intervention to reshape the cranial defect in addition to correcting cosmetic issues. Fronto-orbital advancement is one of the preferred treatment methods in infants older than 6 months. ${ }^{13}$

Although imaging modalities like CT and MRI combined with postprocessing software can provide adequate visualization of this pathology, there are notable limitations of the bidimensional view, and it is not unusual for surgeons to find different anatomical relationships intraoperatively. ${ }^{2}$

The use of a physical hybrid model and AR in planning the operative technique involved in treating metopic craniosynostosis is particularly noteworthy. The preliminary results we report here support the utility of this mixed platform as an introductory adjunct to traditional surgical training.

The use of this kind of preoperative planning tool can reduce errors, maximize sensitivity in demonstrating skills, and improve the learning curve in neurosurgeons in training before they undertake the real surgery. In addition, this method is a useful tool allowing rich 3D visualization (with tactile feedback) that can be applied to patient education and surgical decision-making. The hybrid model we present here allowed a detailed discussion of the aspects of the surgical approach by presenting different consistencies and resistances of the lifelike material and enabling the prediction of bilateral orbitotomy measurements with millimetric accuracy. ${ }^{12}$

We believe that hybrid models allow for enhanced insight into the underlying anatomy since they supply comprehensive 3D imagery and tactile feedback for each abstraction. Compared with traditional imaging and other methods of advanced imaging visualization such as 3D computer models, the 3D-printed models, as delineated by Wake et al., individually join multisensory inputs of touch and vision, leading to improved spatial conceptualization compared with simple 3D anatomical images viewed as a computer model. ${ }^{16}$ Some previous studies have demonstrated the possibilities of AR combined with simulation, exercises, and feedback modes for the improvement of physical abilities. ${ }^{5}$

This new AR technology coupled to mobile devices as an app presented important advantages. It is patient specific, provides reliable and multiangular visualization (with rich geometrical representation), shows freehand navigation, is scalable, and enables rotation of the patient's image and zooming in and out to any size. Furthermore, it is possible to visualize from a "complete" reconstruction (showing many structures) to "parts" of the anatomical model (Fig. 5). After the development phase, widespread use can be available at a low cost. Finally, this gadget can provide the basis for a robust and rich multidisciplinary discussion and can be considered a resource optimizer in preoperative surgical planning.

Although the efficacy evaluation of the hybrid model was done by the case patient's surgeons, one limitation of this study was that the questionnaire evaluation was not performed for the hybrid model but only for the AR tool. Besides that, this study did not include a detailed cost analysis. The estimated cost of the hybrid model is $\$ 1700.00$, but this model is still in an experimental phase and is not yet commercially available. The AR mobile platform is in the same development stage; however, at this moment it is not possible to estimate its possible costs.

This report not only presents what is to our knowledge the first description of a mixed platform that combines AR on a mobile app with a 3D physical hybrid model for craniosynostosis preoperative planning, but also reinforces future perspectives. Notably, this method should be useful for abbreviating the learning curve during the training of young surgeons while minimizing the complications of technical errors. Such models can be applied in a revolu- 

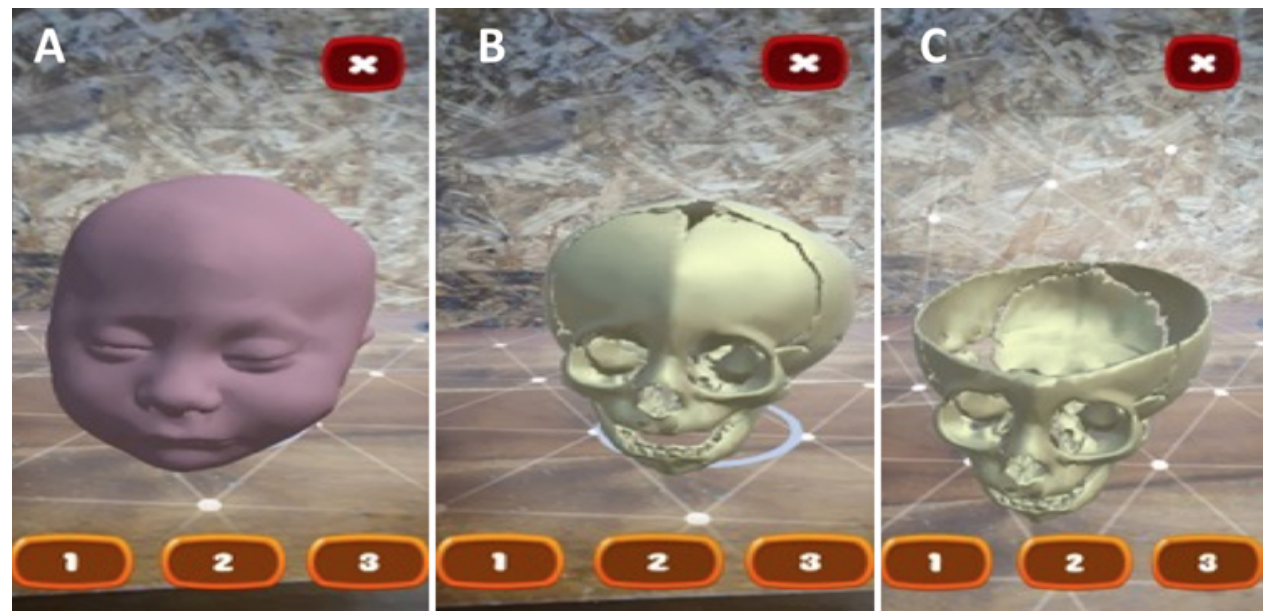

FIG. 5. The 3 layers of visualization in the AR app. A: AR image of patient face in CT scan. B: Whole skull format. C: Possibilities of AR skull creation.

tionary and safe manner to the training of neurosurgeons in performing other operative techniques used in the correction of craniofacial malformations.

Our study is not without limitations. This was the first attempt to develop a hybrid model, and the sample size was small. The Likert scale assessment tool, although subjective, represented the opinions of senior experts in the field of craniofacial surgery and neurosurgery.

\section{Conclusions}

The association of physical hybrid model technology with the application of AR can provide a highly effective training environment by facilitating preoperative planning while highlighting notable perspectives in medical education. The remarkable realism of this method significantly enhances the teaching of surgical anatomy and operative strategies in the neurosurgical field, especially during residency training, and the 3D modeling technology may provide a complementary educational tool in any preoperative surgical planning, enhancing the safety of the procedure and contributing to a better outcome.

\section{Acknowledgments}

We thank Gustavo Zagatto and Kid Mendes for the creation of the virtual model for the AR device and hybrid model and Tadeu Menegatti Mendes for his assistance in preparing the pictures. We also thank Samsung and Medtronic companies and the EDUCSIM Institute for scientific support and Fabiano Ribeiro Souza Carvalho, Eduardo Conejo, and Luis Guilherme Selber for their important collaboration in this project. Finally, we thank Pedro Augusto Marques de Lima for making the video.

\section{References}

1. Christian EA, Imahiyerobo TA, Nallapa S, Urata M, McComb JG, Krieger MD: Intracranial hypertension after surgical correction for craniosynostosis: a systematic review. Neurosurg Focus 38(5):E6, 2015

2. Coelho G, Chaves TMF, Goes AF, Del Massa EC, Moraes O, Yoshida M: Multimaterial 3D printing preoperative planning for frontoethmoidal meningoencephalocele surgery. Childs Nerv Syst 34:749-756, 2018
3. Coelho G, Warf B, Lyra M, Zanon N: Anatomical pediatric model for craniosynostosis surgical training. Childs Nerv Syst 30:2009-2014, 2014

4. Cornelissen MJ, Loudon SE, van Doorn FE, Muller RP, van Veelen MC, Mathijssen IM: Very low prevalence of intracranial hypertension in trigonocephaly. Plast Reconstr Surg 139:97e-104e, 2017

5. Dede C: Immersive interfaces for engagement and learning. Science 323:66-69, 2009

6. Dempsey RF, Monson LA, Maricevich RS, Truong TA, Olarunnipa S, Lam SK, et al: Nonsyndromic craniosynostosis. Clin Plast Surg 46:123-139, 2019

7. Han W, Yang X, Wu S, Fan S, Chen X, Aung ZM, et al: A new method for cranial vault reconstruction: augmented reality in synostotic plagiocephaly surgery. J Craniomaxillofac Surg 47:1280-1284, 2019

8. Hicdonmez T, Parsak T, Cobanoglu S: Simulation of surgery for craniosynostosis: a training model in a fresh cadaveric sheep cranium. Technical note. J Neurosurg 105 (2 Suppl):150-152, 2006

9. López Cabrera MV, Valdez García JE: Virtual reality in residents training. Ann Eye Sci 4:27, 2019

10. Meola A, Cutolo F, Carbone M, Cagnazzo F, Ferrari M, Ferrari V: Augmented reality in neurosurgery: a systematic review. Neurosurg Rev 40:537-548, 2017

11. Rice DP: Craniofacial Sutures. Development, disease and treatment. Front Oral Biol 12:xi, 2008

12. Si WX, Liao XY, Qian YL, Sun HT, Chen XD, Wang Q, et al: Assessing performance of augmented reality-based neurosurgical training. Vis Comput Ind Biomed Art 2:6, 2019

13. Taşkapılığlu MO, Ocakoğlu G, Kaya S, Baykal D, Yazıcı Z: Statistical shape analyses of trigonocephaly patients. Childs Nerv Syst 36:379-384, 2020

14. Vinchon M: The metopic suture: natural history. Neurochirurgie 65:239-245, 2019

15. Vu HL, Panchal J, Parker EE, Levine NS, Francel P: The timing of physiologic closure of the metopic suture: a review of 159 patients using reconstructed 3D CT scans of the craniofacial region. J Craniofac Surg 12:527-532, 2001

16. Wake N, Rosenkrantz AB, Huang R, Park KU, Wysock JS, Taneja SS, et al: Patient-specific 3D printed and augmented reality kidney and prostate cancer models: impact on patient education. 3D Print Med 5:4, 2019

17. Weinzweig J, Kirschner RE, Farley A, Reiss P, Hunter J, Whitaker LA, et al: Metopic synostosis: Defining the temporal sequence of normal suture fusion and differentiating 
it from synostosis on the basis of computed tomography images. Plast Reconstr Surg 112:1211-1218, 2003

18. Whitaker LA, Bartlett SP, Schut L, Bruce D: Craniosynostosis: an analysis of the timing, treatment, and complications in 164 consecutive patients. Plast Reconstr Surg 80:195-212, 1987

19. Wójcicki P, Prudel B: Trigonocephaly: Long-term results after surgical correction of metopic suture synostosis. Adv Clin Exp Med 28:625-635, 2019

\section{Disclosures}

The authors report no conflict of interest concerning the materials or methods used in this study or the findings specified in this paper.

\section{Author Contributions}

Conception and design: Figueiredo, Coelho, Rabelo, Vieira, Mendes, Zagatto, Santos de Oliveira, Yoshida, de Souza, Teixeira. Acquisition of data: all authors. Analysis and interpretation of data: Figueiredo, Coelho, Rabelo, Raposo-Amaral, Yoshida, de Souza, Fagundes, Teixeira. Drafting the article: Figueiredo, Coelho, Rabelo, Raposo-Amaral, Yoshida, Teixeira. Critically revising the article: Figueiredo, Coelho, Rabelo, Raposo-Amaral. Reviewed submitted version of manuscript: Figueiredo, Coelho, Rabelo. Statistical analysis: Figueiredo, Coelho, Rabelo. Administrative/technical/material support: Figueiredo, Coelho. Study supervision: Figueiredo.

\section{Supplemental Information \\ Videos}

Video 1. https://vimeo.com/385974104.

\section{Correspondence}

Eberval Gadelha Figueiredo: University of São Paulo, Brazil. ebgadelha@yahoo.com. 